\title{
Introduction to the case studies
}

The approach taken to human rights and rights promotion in the following case studies flows from the themes raised in Part I. Two simple ideas here are primary. The first is that notions of human rights, at their most fundamental level of significance, are one way of dealing with the perennial problems of the systemic infliction of suffering, particularly gross suffering, as a mechanism or a function of political organisation. That is, human rights practices are one way of articulating and working against the harm we do each other, and of encouraging political contexts of non-injury and mutual respect. We account for and recognize harm in different ways - notions of human rights are not a metalanguage, but they can operate as a way into the complexities and confusions of particular cases and broader patterns of injury as well as, in very general terms, asserting the value of people. The second idea informing the approach taken in the case studies is that 'dialogue' or 'conversation' - paying people and the pattern of their lives the fundamental respect of listening to them and being engaged with them - is at the core of both respecting and promoting human rights.

A number of themes follow from these two simple points of beginning. To take part in serious exchanges on difficult social and political problems often involves a preparedness to look at yourself and your tools differently. Thus, critical reflection on the methods, outcomes and assumptions of the human rights models and practices that are being promoted is essential to this approach - not in order to abandon traditions of rights practices but to recognise that they do not constitute a fixed or necessarily emancipatory truth. One of the purposes of the earlier chapters, in this context, is to provide some critical distance from the ways we tend to think about and work with human rights in the international domain. In stepping back from human rights as a definitive model of state and individual we meet again the complexity of rights as a series of questions about patterns of injury which are recurrent as well as embedded in and given meaning by concrete circumstances of time and place.

Thus, rather than being primarily an evangelical task of 'truth-bearing', or an assertion of the inevitable 'rightness' of a particular model of government, the promotion of human rights may demand long-term engagement with particular institutions or knots of social practice - with mechanisms for constructing community - across and between cultures. Response to abuse is part of a long and slow conversation between and across cultures on the nature of political community and the place of injury within it. In practical terms, efforts to change violent or injurious social practices require that cultural cosmologies not be dismissed wholesale as incompatible with the emergence of a working rights practice but be taken seriously as means of working against abuse or as potential grounds for the evolution of such practices. Moreover, it may be that 
responses to particular patterns of abuse that are effective over the long term emerge essentially in interaction with the people who live the situation to hand (whether that is ourselves or others) and the social and political orders they inhabit. It is this work which gives substance to and underpins international treaties and declarations.

The following three case studies look at quite different situations. The first considers an event: the Tiananmen Square massacre in China in 1989. This case study looks at the way a language of indignation that draws significantly on Lockean models of the state, political community and human rights may hinder understanding of and response to particular situations of abuse - even when that situation, in this case a textbook example of the grave abuse of citizens by their own government in the heart of the state, seems particularly suited to those models. The question of notions of 'human' rights versus citizen's rights is also touched on briefly here.

The second case study discusses briefly the last twenty-five years of East Timor's history, focusing on the context of Indonesia's violent occupation and the forced pace of nation building, and touching on some of the issues of peace builidng in the new state. The discussion underlines the persistent failure to engage with the grassroots dynamics of circumstances in East Timor in the creation and perpetuation of a pattern of severe and embedded abuse. That failure to pay attention to concrete circumstances marked the 'realism' of the prevailing international attitudes on East Timor; to what extent might it also characterise the current liberal approaches?

The third case study, which looks at the 'place' of Indigenous Australians within Australian political life, returns to a liberal rights focus - in this case not involving the language of international rights talk but rather concerning the ideals and practices of democratic political community. This study examines the politics of health in particular as a means of exploring how the construction of consent and participation as the basis of the state have worked to exclude Indigenous people. By discussing one obdurate complex of abuse within a wealthy western democratic state, chapter 6 again reflects on some of the tools the liberal democratic states bring to understanding and responding to abusive situations. It explores the notion of participation, as one that is central to liberal constructions of citizenship and also to the approach taken here, and considers what is in this case the essentially political nature of 'social and economic' rights.

The pattern of difference and similarity among the three case studies at best allows the studies to talk to each other, clarifying some points and opening up ambiguities in others. Despite the range of difference among the cases, it is important to note that they certainly do not cross the whole gamut of forms of abuse. Even across the range of three case studies, key aspects of the argument, particularly the emphasis on human rights promotion as a process of mutual 
communication rather than a message, apply differently. This emphasis is likely to speak directly and fully to some kinds of abuse and in practical terms only highly contextually and indirectly to certain other cases, where any possibility of effective dialogue has long passed or not yet arisen.

The topics addressed by the three case studies are far too complex to be dealt with in depth by a single chapter on each. In this sense the studies cannot bear out the argument made here in that they do not give to each set of circumstances the level of attention the argument would suggest is necessary. Nor does each chapter set itself the Promethean task of resolving the problem it discusses; rather it points, where possible, to a shift of emphasis from which resolutions may in time emerge. 